\title{
Sudden Wilt of Melons in Southern Israel: Fungal Agents and Relationship with Plant Development
}

\author{
S. Pivonia, Arava Research and Development, Sapir Center 86825, Israel; R. Cohen, Department of Vegetable \\ Crops, Agricultural Research Organization, Newe Ya'ar Research Center, P.O. Box 1021, Ramat Yishay 30095, Is- \\ rael; U. Kafkafi, Department of Field Crops, The Hebrew University of Jerusalem, Faculty of Agricultural, Food \\ and Environmental Sciences, Rehovot 76100, Israel; I. S. Ben Ze'ev, Ministry of Agriculture and Rural Develop- \\ ment, Plant Protection and Inspection Services, P.O. Box 78, Bet Dagan 50250, Israel; and J. Katan, Department of \\ Plant Pathology and Microbiology, The Hebrew University of Jerusalem, Faculty of Agricultural, Food and Envi- \\ ronmental Sciences, Rehovot 76100, Israel
}

\begin{abstract}
Pivonia, S., Cohen, R., Kafkafi, U., Ben Ze'ev, I. S., and Katan, J. 1997. Sudden wilt of melons in southern Israel: Fungal agents and relationship with plant development. Plant. Dis. 81:12641268.

Fungi belonging to five genera, Monosporascus sp., Pythium aphanidermatum, Rhizoctonia solani, Olpidium sp., Fusarium solani, and $F$. proliferatum, were the species most frequently isolated from the root systems of wilted melon. Diseased plants were collected from 24 fields in the northern and central Arava region of southern Israel during the fall seasons of 1994 and 1995. In pathogenicity tests conducted under field conditions, in artificially inoculated microplots, the first wilt symptoms were observed at various stages of fruit maturation. High mortality levels (73 to 97\%) were recorded for inoculation combinations in which Monosporascus sp. was involved. Inoculations with the other fungi listed resulted in lower incidences of wilt. The combination of $F$. solani and $P$. aphanidermatum resulted in higher mortality than that caused by each pathogen alone. Monosporascus sp. seems to be the primary pathogen, although other fungi could also induce wilt. The dry weight of plants grown in naturally infested soil ceased to accumulate 33 days after transplanting, in contrast to plants grown in methyl bromide-treated soil. At this stage, the first wilt symptoms were observed. Fruit load affected wilt incidence. At the end of the growing season, $98 \%$ mortality was recorded for plants having the normal fruit load (2.5 fruits per plant) compared with 75 and $12 \%$ for plants that had their fruits thinned to one or zero per plant, respectively.
\end{abstract}

Sudden wilt of melon (Cucumis melo L.), also known as melon collapse and vine decline, is a worldwide problem, mainly in arid and semiarid regions (13). This disease is a major problem in the arid Arava region of southern Israel. The Arava valley is a part of the Syrian-African rift valley. Due to high prevailing temperatures, this region became the main out-of-season melon growing area for the local market and for export. The disease causes a rapid wilt of plants bearing mature fruits and may result in a total loss of the crop. Soil disinfestation by fumigation with methyl bromide (MB) is the common treatment used in the Arava to control this disease (21). Growing melons as a monoculture

Corresponding author: R. Cohen

Tel.: 972-4-9539515, Fax: 972-4-9836936

Contribution from the Agricultural Research Organization, The Volcani Center, Bet Dagan, Israel. No. 2047-E 1997 series.

Accepted for publication 24 July 1997.

Publication no. D-1997-0902-03R

(C) 1997 The American Phytopathological Society crop, without crop rotation and without MB fumigation between the growing seasons, may result in severe cases of sudden wilt. The causal agent of this phenomenon in Israel has not been identified in all cases. Several soilborne pathogens, such as Fusarium solani (Mart.) Sacc. f. sp. cucurbita W.C. Snyder \& H.W. Hans, F. equiseti (Corda) Sacc., Macrophomina phaseolina (Tassi) Goid, and Monosporascus eutypoides (Petrak) von Arx have been isolated from such wilted plants in various regions in Israel (5,9,17-19).

In Israel, field trials and inoculation experiments conducted by Reuveni et al. (19) showed that Monosporascus eutypoides was a primary agent of melon collapse in the Jordan Valley, which is a hot and arid region. Similar phenomena have been reported from the United States, Japan, Tunisia, and Spain $(6,11,12,16,22)$. Monosporascus cannonballus Pollack \& Uecker was reported as the main pathogen in the southern part of the United States (12-15); while in the northeastern states, vine decline was associated with Fusarium wilt and cucumber mosaic virus (CMV) (25). Acremonium sp. was isolated from the roots of wilted melon from the Mediterra- nean coastal areas of Spain and various regions in California, and was associated with collapse symptoms $(1,6)$. Monosporascus sp. was also isolated from a relatively low percentage of wilted melons in the same areas of Spain but failed to induce wilt symptoms by artificial inoculation (6).

Observations in melon fields in Israel show that the severity of the disease may increase under certain environmental conditions. It is common in the Arava region that the melon crop may be totally destroyed in late summer (which is a short growing season when temperatures are high); whereas the following melon crop in the same field in the spring (when temperatures are lower) is unaffected or less severely affected by wilt. Significant differences in sudden wilt incidence between the spring and late summer growing seasons were observed during an evaluation of 15 melon genotypes tested in naturally infested soil and harvested in May, September, and November (2). Generally, the highest disease scores were obtained in the late summer growing season compared with spring and fall. This was interpreted as a genotype by environment interaction, which was found to be highly significant (2), or as a result of enhanced activity of thermotolerant microorganisms. Plant response to this disease seems to be influenced by fruit maturation.

The purposes of this research were: (i) to identify the potential agent(s) of melon sudden wilt in the Arava region of southern Israel; (ii) to estimate the agents' relative importance to disease severity, alone and in combinations; and (iii) to quantify the relationship of dry weight accumulation and fruit load to disease development. Brief reports with partial results has been published $(17,18)$.

\section{MATERIALS AND METHODS}

Isolation of fungi from the roots of wilted plants. Wilted melon plants were collected from 24 melon fields (10 plants per field) in the northern and central Arava during the autumn growing seasons (planting in August-September, harvesting in November) of 1994 and 1995. All fields 
from which plants were collected had been fumigated with $\mathrm{MB}$ prior to the growing season. All parts of the root tissue were sampled for the determination of their infection with fungi. Roots of wilted plants were excavated with the aid of a shovel at the early stages of plant collapse. Roots of each plant were plated on five petri plates. Nine segments of the taproot were plated on three plates, and two additional plates were plated with six segments of secondary and tertiary roots. Brown to black root lesions were observed on roots later found to be infected with Rhizoctonia, and small and light brown lesions were observed on roots infected with Pythium aphanidermatum. No symptoms could be detected on roots infected with Monosporascus. Root tissues were surface-disinfested for $1 \mathrm{~min}$ in $1 \% \mathrm{NaOCl}$, rinsed twice in sterile water, and plated on potato dextrose agar (PDA, Difco, Detroit, MI) medium amended with chloramphenicol (Sigma, C-0378) at 250 $\mathrm{mg} / \mathrm{liter}$. For further verification of identity of certain fungi, additional media were used in certain cases: water agar (2\%), peptone-PCNB Fusarium selective medium (3), and asparagine glucose selective medium for Pythium (20). Root colonization by Olpidium sp. was detected with the aid of a light microscope. Roots and rootlets were thoroughly washed under running tap water, cleared with $10 \% \mathrm{KOH}$, acidified in $1 \% \mathrm{HCl}$, and stained with boiling $0.05 \%$ trypan blue (8).

Preparation of inoculum and pathogenicity tests. Plastic cylinders with open ends, $30 \mathrm{~cm}$ diameter and $35 \mathrm{~cm}$ high, were filled with field soil and buried flush with the soil level. Plot beds were covered manually with plastic sheets (Polyon, Kibbutz Barkai, Israel), and the edges were buried in trenches to a depth of $20 \mathrm{~cm}$. MB (Dead Sea Bromine Group, Be'er Sheva, Israel) was applied at a rate of $50 \mathrm{~g} / \mathrm{m}^{2}$ by the hot gas method (7) through the drip irrigation pipes that had been placed on the beds before tarping. After fumigation, the soil content of each cylinder (microplot) was mixed with inoculum of fungi isolated from wilted melon plants prepared as follows: Isolates of Monosporascus sp. were grown for 10 days on PDA at $27^{\circ} \mathrm{C}$. The content of one petri dish was blended with $100 \mathrm{ml}$ of sterile water and mixed with 10 liters of MB-treated soil. In addition, $5 \mathrm{~g}$ of a mixture of soil and inoculated oats, prepared according to Mertely et al. (15), was added to the same soil. $F$. solani and $F$. proliferatum (six and three isolates, respectively) were grown for 1 week on PDA at $27^{\circ} \mathrm{C}$. Conidial suspension was prepared by flooding the plates with $10 \mathrm{ml}$ of distilled water and scraping the medium surface with a glass. The inoculum consisted of $100 \mathrm{ml}$ of a mixture of conidial suspensions of the isolates of each species, adjusted to $10^{6} \mathrm{CFU} / \mathrm{ml}$ (mainly conidia), and mixed with 10 liters of MB-treated soil.

$P$. aphanidermatum was grown on PDA at room temperature for 3 days. Four $1 \times 1$ $\mathrm{cm}$ PDA squares from a culture were cut out and placed in an empty petri dish. Leaves of St. Augustine grass, previously boiled for $30 \mathrm{~min}$ in water, were placed on the PDA squares and incubated at room temperature for 2 days, after which $30 \mathrm{ml}$ of sterile water was added to the dish (20). The contents of several such dishes containing zoospores and hyphae with zoospores were combined afterward and incubated for an additional 2 days at room temperature in an Erlenmeyer flask. One week after planting, $100 \mathrm{ml}$ of fungal suspension at a concentration of $5 \times 10^{4} \mathrm{CFU}$ (mainly zoospores) was drenched at the base of the transplant stem in each cylinder. This was sufficient to moisten the root system of the transplant.

Melon roots infected with Olpidium sp. were thoroughly washed under running tap water and then incubated for $30 \mathrm{~min}$ in sterile water (E. Hadar, personal communication) into which zoospores were released. The inoculum source was tested for possible presence of other pathogens and was found to be free of Pythium and melon necrotic spot virus (MNSV). Each plant was inoculated prior to transplanting by dripping $4 \mathrm{ml}$ of the zoosporic suspension ( 2 to $4 \times 10^{6}$ spores per $\mathrm{ml}$ ) onto its roots. Inoculations combining two species of fungi were done by mixing the two inocula with the same 10 liters of soil, using for each species its particular form of inoculation. Infested soil samples from two fields with a history of disease (at Ein Yahav and Ein Tamar) were used as naturally infested controls. MB-fumigated soil was used as an uninoculated control.

Melon plants were transplanted, three per cylinder, each treatment consisting of 10 cylinders. The field around the cylinders was planted with melons at a stand of 12,000 double plants per hectare. Irrigation and fertilization were according to the recommendations for the Arava region.

Two pathogenicity tests were conducted, the first in the late summer of 1995, transplanted on 16 July and harvested in midSeptember. Average temperatures at planting were 25 and $40^{\circ} \mathrm{C}$ night and day, respectively, and 21 and $37^{\circ} \mathrm{C}$ at harvest. The second test was transplanted on 13 February 1996 and harvested at the beginning of May 1996. Average night and day temperatures at planting were 11 and $21^{\circ} \mathrm{C}$, respectively, and at harvest were 25 and $36^{\circ} \mathrm{C}$. In both tests, plant roots were sampled 3 weeks after the inoculation and plated on PDA in order to determine the efficacy of the inoculation.

Dry weight accumulation in foliage and fruit. In this trial, melon plants (cv. Arava) were grown in naturally infested soil and in MB-treated soil (control) at the Ein Tamar Experiment Station. Plants were transplanted on 15 September 1995. The average daily temperature during September, October, and November 1995 were 25 to $38^{\circ} \mathrm{C}, 20$ to $33^{\circ} \mathrm{C}$, and 15 to $28^{\circ} \mathrm{C}$, respectively. Plants were uprooted for weighing at the following development stages: male flowering, female flowering, $500 \mathrm{~g}$ of fruit, beginning of fruit netting, color breaking, and early maturation, corresponding to $14,22,33,41,50$, and 55 days after transplanting, respectively. Seven plants per treatment were sampled at each stage; the foliage was separated from the fruits, dried at $70^{\circ} \mathrm{C}$ for $48 \mathrm{~h}$, and weighed. Fruits were weighed twice, 33 and 50 days after transplanting. In addition, roots of wilted plants were plated on PDA for the identification of the pathogen, the experimental plots were evaluated for wilt incidence, and the dates of collapse were recorded for each treatment.

Effect of fruit load on disease severity. The effect of fruit load on disease severity was tested in a trial conducted in a field with a history of sudden wilt at Ein Tamar Experiment Station. Fruits were removed 30 days after transplanting, when the first wilt symptoms were observed. New fruits were removed once a week until the completion of the experiment. Disease development (number of wilted plants), was evaluated 46, 53, and 61 days after transplanting, and root samples from wilted plants were plated on PDA for identification of the pathogen. Comparison was made among plants with three levels of fruit load: zero fruits, one fruit, and untreated plants with an average of 2.5 fruits per plant. Each treatment was applied to 50 plants, i.e., 10 plants grown on a 5-m row with five replicates.

Statistical analysis. The significance of differences $(P=0.05)$ in the pathogenicity tests and in the fruit load trial were determined by the Einot-Gabriel range test (4). Disease incidence data were arcsine-transformed before analysis. Selected treatments were thereafter compared by contrast $t$ test. Values of foliage dry weight accumulation are accompanied by SE values.

\section{RESULTS}

Fungi isolated from wilted melon roots. Fungi belonging to five genera were

Table 1. Fungi isolated from melon plants showing collapse during the fall seasons of 1994 and $1995^{\mathrm{z}}$

\begin{tabular}{lcc}
\hline Fungi & $\begin{array}{c}\text { Northern } \\
\text { Arava }\end{array}$ & $\begin{array}{c}\text { Central } \\
\text { Arava }\end{array}$ \\
\hline $\begin{array}{l}\text { Monosporascus } \mathrm{sp} . \\
\text { Pythium }\end{array}$ & 35 & 28 \\
$\quad$ aphanidermatum & 94 & 71 \\
$\begin{array}{l}\text { Rhizoctonia solani } \\
\text { Olpidum sp. }\end{array}$ & 41 & 28 \\
$\begin{array}{l}\text { Fusarium (solani and } \\
\text { proliferatum) }\end{array}$ & 18 & 86 \\
\hline
\end{tabular}

${ }^{\mathrm{z}}$ Values are proportion (\%) of the fields from which indicated fungi were isolated. Wilted plants (10 from each field) were collected from 17 fields in the northern Arava and 7 fields in the central Arava. 
those most frequently isolated from the root systems of wilted melon plants collected from 24 fields in the northern and central Arava during the fall seasons of 1994 and 1995 (Table 1). All fields had been fumigated with $\mathrm{MB}$ in preparation for the growing season. Therefore, the crop in each field developed normally and wilt incidence was usually lower than $5 \%$. The five most frequent genera of fungi were present in both regions, albeit in different proportions. Pythium aphanidermatum and Fusarium (mostly solani and proliferatum) were very common (in 71 to $100 \%$ of fields) in both regions. Olpidium sp. was substantially more common in the central Arava $(86 \%)$ than in the northern Arava (18\%). Monosporascus sp. accounted for only 28 and $35 \%$ of the isolations in the northern and central Arava, respectively (Table 1). Monosporascus was the main pathogen isolated from two fields at the Experiment Stations of Ein Tamar (northern Arava) and Ein Yahav (central Arava), in which melons had been grown without MB fumigation for several seasons and wilt incidence was 73 to $90 \%$ (Tables 2 and 3). Fungi such as Acremonium spp., Alternaria spp., Aspergillus spp., Chaetomium spp., Myrothecium roridum, Nigrospora sp., Trichoderma spp., Fusarium oxysporum, F. equiseti, and Helmintho- sporium spp. were also isolated, but they were substantially less frequent than the species listed in Table 1, and their pathogenicity to melon plants was not tested.

Pathogenicity tests. In the fall of 1995 , plants wilted and collapsed in the inoculated microplots starting in mid-September, when fruits were at various stages of maturity. Mortality percentages were recorded 70 days after planting. When plants were inoculated with a single species of pathogen, Monosporascus sp. was the most virulent, producing $73 \%$ mortality, significantly higher than $P$. aphanidermatum, $F$. solani, Olpidium sp., and $F$. proliferatum (Table 2). The ripening of fruits on plants inoculated with Olpidium sp. was somewhat delayed compared with those in the other treatments, while wilt was comparable with that in the uninoculated control.

Several combinations of inoculations resulted in high disease incidence (Table 2). The highest mortality levels were recorded for inoculation combinations involving Monosporascus sp. (80 to $97 \%$ ), but they did not significantly differ from inoculation with Monosporascus alone (73\%). Disease incidence in naturally infested soils from which Monosporascus was the pathogen most frequently isolated was also high (73 to $90 \%$ ). However, the contrast

Table 2. Response of melon plants to inoculation with fungi isolated from wilted plants or growth in infested soil from two locations ${ }^{y}$

\begin{tabular}{lc}
\hline Inoculation source & Wilt incidence (\%) \\
\hline Monosporascus sp. + Fusarium proliferatum & $97 \mathrm{a}^{\mathrm{z}}$ \\
Monosporascus sp. + Fusarium solani & $97 \mathrm{a}$ \\
Naturally infested soil - EinYahav & $90 \mathrm{a}$ \\
Monosporascus sp. + Pythium aphanidermatum & $80 \mathrm{a}$ \\
Monosporascus sp. + Olpidium sp. & $80 \mathrm{a}$ \\
Monosporascus sp. & $73 \mathrm{ab}$ \\
Naturally infested soil - Ein Tamar & $73 \mathrm{ab}$ \\
Fusarium solani + Pythium aphanidermatum & $57 \mathrm{abc}$ \\
Pythium aphanidermatum + Olpidium sp. & $30 \mathrm{abcd}$ \\
Pythium aphanidermatum & $23 \mathrm{~cd}$ \\
Fusarium solani + Fusarium proliferatum & $17 \mathrm{~d}$ \\
Fusarium solani & $17 \mathrm{~d}$ \\
Olpidium sp. & $3 \mathrm{~d}$ \\
Fusarium proliferatum & $0 \mathrm{~d}$ \\
Fusarium solani + Olpidium sp. & $0 \mathrm{~d}$ \\
Methyl bromide-treated soil, noninoculated & $0 \mathrm{~d}$ \\
\hline
\end{tabular}

y The trial was conducted under field conditions in fall 1995. Planting on 16 July 1995. Fruit maturity at mid-September 1995. Disease incidence was recorded 70 days after planting.

${ }^{\mathrm{z}}$ Values with the same letter are not significantly different $(P=0.05)$.

Table 3. Response of melon plants to inoculation with fungi isolated from wilted plants or growth in infested soil from two locations ${ }^{y}$

\begin{tabular}{lc}
\hline Inoculation source & Wilt incidence (\%) \\
\hline Naturally infested soil - Ein Tamar & $90 \mathrm{a}^{\mathrm{z}}$ \\
Monosporascus sp. & $88 \mathrm{a}$ \\
Naturally infested soil - Ein Yahav & $80 \mathrm{a}$ \\
Pythium aphanidermatum + Fusarium solani & $72 \mathrm{ab}$ \\
Pythium aphanidermatum & $54 \mathrm{ab}$ \\
Fusarium solani & $16 \mathrm{~b}$ \\
Methyl bromide-treated soil, noninoculated & $12 \mathrm{~b}$ \\
\hline
\end{tabular}

y The trial was conducted under field conditions in spring 1996. Planting on 13 February 1996. Fruit maturity at beginning of May 1996. Disease incidence was recorded 94 days after planting.

${ }^{\mathrm{z}}$ Values with the same letter are not significantly different $(P=0.05)$. test comparing Monosporascus versus combinations with other fungi showed significantly greater wilt incidence for combinations $(P=0.013)$. This was also the case for the combination of $F$. solani + $P$. aphanidermatum $(P=0.0001)$.

The results obtained in the first trial were essentially confirmed in the second pathogenicity test, conducted in the spring of 1996 (Table 3). Monosporascus-inoculated plants and plants grown in naturally infested soil exhibited the highest wilt incidence, ranging from 80 to $90 \%$, similar to the incidence (73 to $90 \%$ ) in naturally infested soil. $P$. aphanidermatum and the combination of Pythium with $F$. solani resulted in 54 and $72 \%$ mortality, respectively. Pythium was the only fungus that produced different values for sudden wilt in the second test (54\%), compared with $23 \%$ at the first test. Although $R$. solani was isolated in relatively high percentages from wilted melon plants (Table 1), it failed to induce wilt symptoms in preliminary experiments; thus it was not included in the pathogenicity tests.

Dry weight accumulation in foliage and fruit. The foliage dry weight of plants grown both in MB-fumigated soil and in naturally infested soils accumulated up to 33 days after planting but was 35\% greater in plants grown in fumigated soil. This stage was a turning point at which dry weight accumulation ceased for plants in infested soils but continued at a slower rate for plants in fumigated soil (Fig. 1).

The average fruit dry weight of plants grown in MB-treated soil increased from $64 \mathrm{~g}$ on the thirty-third day to $184 \mathrm{~g} 50$ days after planting, compared with $51 \mathrm{~g}$ and $107 \mathrm{~g}$, respectively, for plants grown in infested soils. The first wilt symptoms were observed in the latter plants 32 to 35 days after planting.

Effect of fruit load on disease severity. The first symptoms of sudden wilt in this experiment were usually associated with fruit maturity. Mortality of $98 \%$ of the untreated plants grown in infested soil, with an average of 2.5 fruits per plant, was observed 61 days after planting. Plants grown in infested soil, but with fruit thinned to one or zero per plant, exhibited wilt incidences of 75 and $12 \%$, respectively (Fig. 2). Monosporascus sp. was the fungus most frequently isolated from the roots of wilted plants in this experiment.

\section{DISCUSSION}

Monosporascus eutypoides has been reported $(5,9,19)$ to be associated with collapse of melons in the Jordan Valley and the Arava of southern Israel. Both regions are arid and characterized by hot climates. Monosporascus sp. was the most frequently isolated from nonfumigated naturally infested soil. However, in MBfumigated soils, it was less frequent than the other fungi, such as $P$. aphanidermatum and Fusarium spp. This apparently 
reflects microbial shift induced by $\mathrm{MB}$ fumigation.

Pathogenicity tests were conducted in order to establish the relative importance of the various fungi isolated from wilted plants to the sudden wilt syndrome of melons in the Arava. Using artificial inoculations, Monosporascus sp. was found to be the single most virulent species involved in the sudden wilt of melons in the Arava region. $P$. aphanidermatum and $F$. solani, although more frequently isolated, were shown to be less important as single pathogens under Arava conditions. Their importance seems to be in their ability to cocolonize the roots of melon plants and to induce wilt to a greater extent than their individual effects, thus contributing to the disease complex.

$F$. proliferatum was not pathogenic by itself or in combination with $F$. solani. $F$. proliferatum is widespread in Israel, where it sometimes causes root rot and stem base rot in various crops (I. S. Ben Ze'ev and E.



DAYS AFTER PLANTING

Fig. 1. Accumulation of dry weight in the foliage of melon plants grown in methyl bromide-treated soil or in infested soil. Bars indicate SE values. Arrow indicates the time at which the first wilt symptoms were observed.

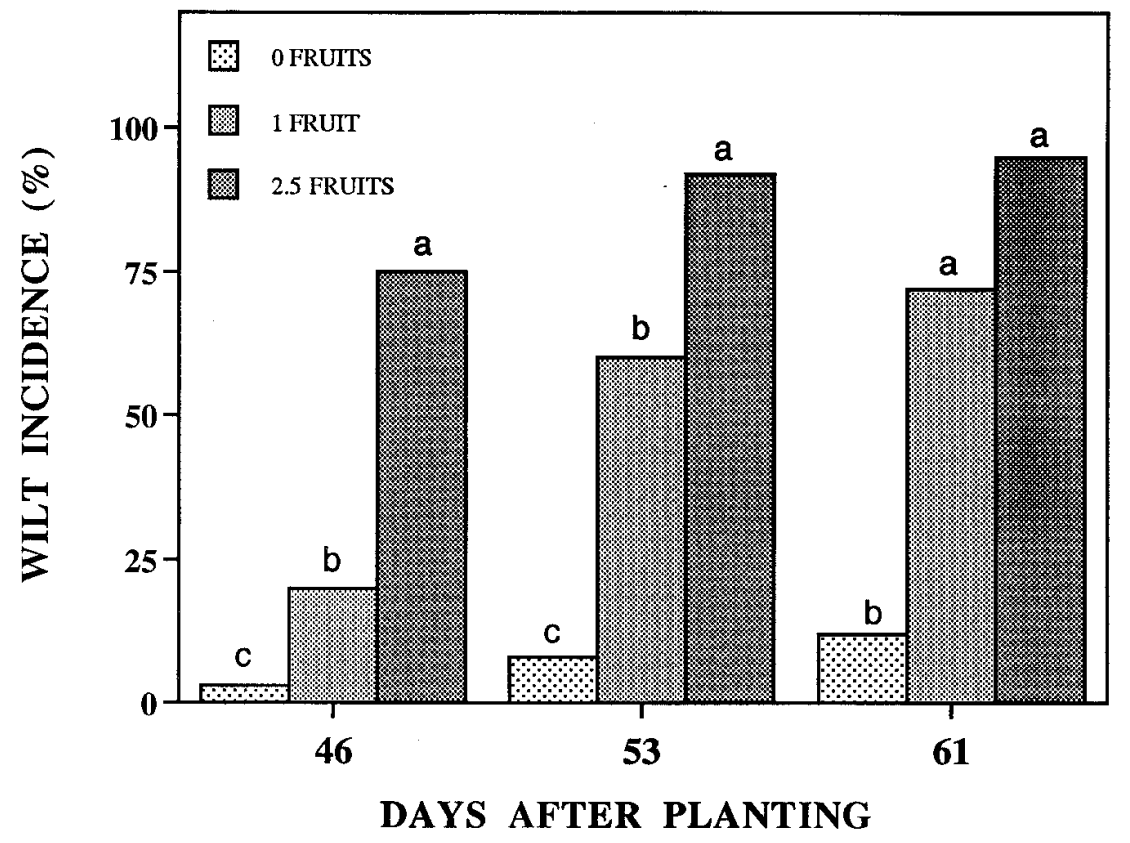

Fig 2. Effect of fruit removal on wilt incidence. Bars (for the same date) with a common letter are not significantly different $(P=0.05)$.
Levy, unpublished). In the second test, conducted in the spring of 1996 (Table 3), $P$. aphanidermatum was capable of inducing wilt symptoms in $54 \%$ of the inoculated plants, compared with $23 \%$ in the fall experiment. These differences between the two experiments might be attributed to the environmental conditions and the length of the growing period.

A variety of organisms has been reported as causal agents of melon collapse in various regions $(5,6,9,12,14)$. It is possible that all of these organisms act similarly by suppressing the root system, finally affecting the balance between sink and source, especially during the period of fruit maturation. Abiotic stresses, such as high temperature, may further aggravate the stress situation.

Foliage dry weight of melon plants ceased to accumulate when the fruits increased in weight. This is in agreement with the reports that reproductive activity, i.e., fruit development, curtails the vegetative growth of plants including cucurbits $(10,24)$. The high demand for water and assimilates by the developing fruit, in addition to the need to continue foliage and root maintenance, can endanger plants with roots damaged by pathogenic organisms. This turning point was observed in the present study around the thirty-third day after planting, when fruit weight was approximately $500 \mathrm{~g}$ (Fig. 1), in plants grown in MB-treated soil, but was more pronounced in plants grown in infested soil. At this stage, a reversible loss of turgor was observed first around midday, and wilt symptoms were detected a few days later. The contribution of fruit maturation to enhanced symptom development was further tested by removing the fruits prior to ripening. Fruit removal may reduce the water stress and, consequently, plant wilting (Fig. 2). Similar results, showing delayed symptoms following fruit removal, were reported in trials conducted in Texas (23).

The presented data show that Monosporascus can be found in plants grown in MB-treated soils in the first growing season after fumigation. This fungus became the primary pathogen in the two experimental field plots after a few seasons of melon growing without MB application. It seems that Monosporascus has an advantage in infesting the soils used for melon monoculture in this particular area.

\section{ACKNOWLEDGMENT}

We thank Ester Hadar for helpful suggestions regarding Olpidium and Pythium studies.

\section{LITERATURE CITED}

1. Bruton, B. D., Davis, R. M., and Gordon, T. R. 1995. Occurrence of Acremonium sp. and Monosporascus cannonballus in the major cantaloupe and watermelon growing areas of California. Plant Dis.79:754.

2. Cohen, R., Elkind, Y., Burger, Y., Offenbach, R., and Nerson, H. 1996. Variation in the response of melon genotypes to sudden wilt. 
Euphytica 87:91-95

3. Dhingra, O. D., and Sinclair, J. B. 1986. Basic Plant Pathology Methods. CRC Press, Boca Raton, FL.

4. Einot, I., and Gabriel, K. R. 1975. A study of the power of several methods of multiple comparisons. J. Am. Stat. Assoc. 70:574-583.

5. Eyal, H., and Cohen, Y. 1986. Sudden wilt in muskmelon: A continuing challenge. (Abstr.) Phytoparasitica 14:251.

6. Garcia-Jimenez, J., Velazquez, M. T., Jorda, C., and Alfaro-Garcia, A. 1994. Acremonium species as the causal agent of muskmelon collapse in Spain. Plant Dis. 78:416-419.

7. Klein, L. 1996. Methyl bromide as soil fumigant. Pages 191-235 in: The Methyl Bromide Issue. C. H. Bell, N. Price, and B. Chakrabarti, eds. John Wiley \& Sons, New York.

8. Koske, R. E., and Gemma, J. N. 1989. A modified procedure for staining roots to detect VA mycorrhizas. Mycol. Res. 92:486505 .

9. Krikun, J. 1985. Observations on the distribution of the pathogen Monosporascus eutypoides as related to soil temperature and fertigation. Phytoparasitica 13:225-228.

10. Leonard, E. R. 1962. Inter-relation of vegetative and reproductive growth, with special reference to indeterminate plants. Bot. Rev. 28:353-410.

11. Martyn, R. D., Lovic, B. R., Maddox, D. A., Germash, A., and Miller, M. E. 1994. First report of Monosporascus root rot/vine decline of watermelon in Tunisia. Plant Dis. 78:1220.

12. Martyn, R. D., and Miller, M. E. 1996. Mono- sporascus root rot and vine decline of muskmelon and watermelon. Pages 18-19 in: Compendium of Cucurbit Diseases. T. A. Zitter, D. L. Hopkins, and C. E. Thomas, eds. American Phytopathological Society, St. Paul, MN.

13. Martyn, R. D., and Miller, M. E. 1996. Monosporascus root rot and vine decline: An emerging disease of melons worldwide. Plant Dis. 80:716-725.

14. Mertely, J. C., Martyn, R. D., Miller, M. E., and Bruton, B. D. 1991. Role of Monosporascus cannonballus and other fungi in root rot/vine decline disease of muskmelon. Plant Dis. 75:1133-1137.

15. Mertely, J. C., Martyn, R. D., Miller, M. E., and Bruton, B. D. 1993. An expanded host range for the muskmelon pathogen Monosporascus cannonballus. Plant Dis. 77:667-673.

16. Miller, M. E., Martyn, R. D., Lovic, B. R., and Bruton, B. D. 1995. An overview of vine decline diseases in melons. Pages 31-35 in: Proc. Cucurbitaceae 94: Evaluation and Enhancement of Cucurbit Germplasm. G. E. Lester and J. R. Dunlap, eds. Gateway Printing, Edinburgh, TX.

17. Pivonia, S., Cohen, R., Katan, J., Ben Zeev, I. S., and Kafkafi, U. 1996. Phytopathological and physiological aspects of sudden wilt of melons in the Arava region. (Abstr.) Phytoparasitica 24:142.

18. Pivonia, S., Cohen, R., Katan, J., Burger, Y. Ben Ze'ev, I. S., Karchi, Z., and Edelstein, M. 1996. Sudden wilt of melons in Southern Israel. Pages 285-290 in: Proc. Eucarpia Meet. Cucurbit Genet. Breed., 6th.
19. Reuveni, R., Krikun, J., and Shani, U. 1983. The role of Monosporascus eutypoides in a collapse of melon plants in an arid area of Israel. Phytopathology 73:1223-1226.

20. Schmitthenner, A. J. 1979. Pythium species: Isolation and identification. Pages 33-36 in: Advances in Turfgrass Pathology: Turfgrass Diseases. P. O. Larsen and B. G. Jogner, eds. Harcourt Brace Jovanovich, Duluth, MN.

21. Ucko, O., Maduel, A., Grinstein, A., and Katan, J. 1992. Combined methods of soil disinfestation for controlling melon collapse with reduced methyl bromide dosages. (Abstr.) Phytoparasitica 20:229-230.

22. Uematsu, S., Hirota, K., Shiruishi, T., Ooizumi, T., Sokiyama, K., Ishikura, I., and Edagawa, Y. 1992. Monosporascus root rot of bottle gourd stock of watermelon caused by Monosporascus cannonballus. Ann. Phytopathol. Soc. Jpn. 58:354-359.

23. Wolff, D. W. 1994. Genotype, fruit load and temperature affect Monosporascus root rot/vine decline symptoms expression in melon (Cucumis melo L.). Pages 280-284 in: Proc. Eucarpia Meet. Cucurbit Genet. Breed., 6 th.

24. Zack, C. D., and Loy, B. 1981. Effect of fruit development on vegetative growth of squash. Can. J. Plant Sci. 61:673-676.

25. Zitter, T. A. 1995. Sudden wilt of melons from northern US perspective. Pages 44-47 in: Proc. Cucurbitaceae 94: Evaluation and Enhancement of Cucurbit Germplasm. G. E. Lester and J. R. Dunlap, eds. Gateway Printing, Edinburgh, TX 